\title{
Magneto-optical properties of bilayer transition metal dichalcogenides
}

\author{
M. Zubair ${ }^{1}$, M. Tahir ${ }^{2}$, and P. Vasilopoulos ${ }^{1}$ \\ ${ }^{1}$ Department of Physics, Concordia University, 1141 Sherbrooke Ouest, Montreal, Quebec H4B 1R6, Canada \\ ${ }^{2}$ Department of Physics, Colorado State University, Fort Collins, CO 80523, USA
}

\begin{abstract}
In transition metal dichalcogenides the spin-orbit interaction affects differently the conduction and valence band energies as functions of $k$ and the band gap is large. Consequently, when a perpendicular magnetic field $B$ is applied the conduction and valence band Landau levels are also different and this leads to a splitting of the interband optical absorption lines in both the absence and presence of an external electric field $E_{z}$. When $B$ and $E_{z}$ are present the peaks in the imaginary part of the Hall conductivity give two distinct contributions of opposite sign to the interband spectrum. The real part of the right- and left-handed interband conductivity, however, retains its two-peak structure but the peaks are shifted in energy and amplitude with respect to each other in contrast with graphene. The response of the intraband conductivity is significantly modified when the Fermi energy $E_{F}$ and the field $B$ are varied. Its optical spectral weight is found to increase with $E_{F}$ in contrast with the decrease observed in graphene. Further, the position and amplitude of the intraband response depends on the field $B$. The absorption peaks vary linearly with $B$ for all fields similar to bilayer graphene for low fields but in contrast with the high-field $\sqrt{B}$ dependence in it.
\end{abstract}

\section{INTRODUCTION}

Two-dimensional materials have attracted a lot of attention due to their applications in spintronics 1 , valleytronics 2 and optoelectronics $\frac{3.4}{2}$. In this regard the group IV transition-metal dichalcogenides (TMDCs) have the form $\mathrm{MX}_{2}(\mathrm{M}=\mathrm{Mo}, \mathrm{W} ; \mathrm{X}=\mathrm{S}, \mathrm{Se})$ are of particular interest due to their valley degree of freedom, large direct band gap $3,5,6$ and strong intrinsic spin-orbit interaction $(\mathrm{SOI})^{\underline{7}, \underline{8}}$. Recently, nanoelectronic devices, such as amplifiers, photodetectors, thin film transistors, and logical circuits $4.9-11$, based on their excellent electronic properties have been experimentally realized. In addition, several properties of TMDC monolayers have been investigated theoretically and experimentally $12-17$ e.g., magneto-optical spectra and magnetotransport.

Layered TMDCs, such as bilayer systems, exhibit a broad range of physical properties and have been extensively studied for applications in catalysis, tribology, electronics, photovoltaics, and electrochemistry $\underline{18-21}$. Also, few layer TMDCs have potential applications in nanoelectronics and nanophotonics. A field-effect transistor has been realized experimentally in a few-layer $\mathrm{MoS}_{2} 22$. Similarly, magnetoelectric effects and valley-controlled spin quantum gates 23 , tuning of the valley magnetic moment 24 , electrical control of the valley-Hall effect 25 , and spin-layer locking effect $\underline{26}$ has been explored in bilayer TMDCs. Most recently, magnetotransport studies of bilayer $\mathrm{MoS}_{2}$ have been carried out 27 . Additionally, a band gap tuning is possible and more easily achievable in bilayer TMDCs than in monolayer TMDCs in the presence of a perpendicular electric field $E_{z} \underline{28,29}$. However, less attention has been paid to the optical properties of bilayer TMDCs in the simultaneous presence of electric and magnetic fields.

In this work we study in detail the effect of magnetic and electric fields on the magneto-optical conductivity of bilayer TMDCs with particular emphasis on the asymmetry between the conduction band $(\mathrm{CB})$ and valence band (VB). Moreover, we assess the effect of the electric field on the band structure with and without magnetic field, and on the magneto-optical conductivities. Also, we compare our results with those for monolayer and bilayer graphene.

We focus on bilayer $\mathrm{WSe}_{2}$ due to recent experimental progress $24,25,30-33$ but our findings are equally pertinent to other bilayer TMDCs, e.g. $\mathrm{MoSe}_{2}$ and $\mathrm{WS}_{2}$. The $\mathrm{WSe}_{2}$ bilayer has much stronger SOI in the conduction $\left(2 \lambda_{c}=30 \mathrm{meV}\right)$ and valence $\left(2 \lambda_{v}=450 \mathrm{meV}\right)$ bands compared to bilayer $\operatorname{MoS}_{2}\left(2 \lambda_{c}=0 \mathrm{meV}\right)$. The bandedge energy difference $E_{\Gamma K}$ between the $\Gamma$ and $K$ points is much smaller than in bilayer $\mathrm{WSe}_{2}$ than in $\mathrm{MoS}_{2} \underline{34,35}$. Therefore, the $\mathrm{CB}$ and $\mathrm{VB}$ edges in bilayer $\mathrm{WSe}_{2}$ lie at the $K$ point. Accordingly, bilayer $\mathrm{WSe}_{2}$ has advantages over the $\mathrm{MoS}_{2}$ when studying its optical properties due to the direct band gap at the $\pm K$ points.

In Sec. II we specify the Hamiltonian and obtain the energy eigenvalues and eigenfunctions with and without magnetic field. In Sec. III we present a general expression for the conductivity $\sigma(\omega)$ and provide numerical results. Conclusions and a summary follow in Sec. IV.

\section{ENERGY SPECTRUM}

In $\mathrm{AB}$ stacked bilayer TMDCs the top layer is rotated with respect to the bottom layer by 180 degrees such that the $\mathrm{S}$ atoms in it sit on top of the $\mathrm{M}$ atoms of the bottom layer. As a result, the effective Hamiltonian for bilayer TMDCs can be constructed from that of the single layer by simply adding the interlayer coupling term $\gamma \frac{36}{}$. Then the one-electron Hamiltonian of bilayer $\mathrm{WSe}_{2}$ near the $K$ and $K^{\prime}$ valleys reads $23,24,26,37$

$$
H^{\tau}=\left(\begin{array}{cccc}
-\xi_{1}^{s \tau} & v_{F} \pi_{-}^{\tau} & \gamma & 0 \\
v_{F} \pi_{+}^{\tau} & \xi_{2}^{s \tau} & 0 & 0 \\
\gamma & 0 & -\xi_{3}^{s \tau} & v_{F} \pi_{+}^{\tau} \\
0 & 0 & v_{F} \pi_{-}^{\tau} & \xi_{4}^{s \tau}
\end{array}\right) .
$$


Here $\tau=1(-1)$ is for the $K\left(K^{\prime}\right)$ valley, $\pi_{ \pm}^{\tau}=\tau \pi_{x} \pm i \pi_{y}$, $\xi_{1}^{s \tau}=\kappa+\tau s \lambda_{v}+s M_{z}-\tau M_{v}, \xi_{2}^{s \tau}=\alpha-\tau s \lambda_{c}-s M_{z}+\tau M_{v}$, $\xi_{3}^{s \tau}=\alpha-\tau s \lambda_{v}-s M_{z}+\tau M_{v}, \xi_{4}^{s \tau}=\kappa+\tau s \lambda_{c}+s M_{z}-\tau M_{v}$ and $\kappa=\Delta+V$ and $\alpha=\Delta-V$ with $\Delta$ the monolayer band gap. Further, $v_{F}=5 \times 10^{5} \mathrm{~m} / \mathrm{s}$ is the Fermi velocity, $V$ is the potential difference between the two layers due to a perpendicular electric field $E_{z}$, and $\lambda$ the strength of the SOI with spins up (down) represented by $s=+1(\uparrow)(s=-1(\downarrow))$. Moreover, $M_{z}=g^{\prime} \mu_{B} B / 2$ is the Zeeman exchange field induced by ferromagnetic order, $g^{\prime}$ the Landé $g$ factor $\left(g^{\prime}=g_{e}^{\prime}+g_{s}^{\prime}\right)$, and $\mu_{B}$ the Bohr magneton ${ }^{38.39} ; g_{e}^{\prime}=2$ is the free electron $g$ factor and $g_{s}^{\prime}=0.21$ the out-of-plane factor due to the strong SOI. The term $M_{v}=g_{v}^{\prime} \mu_{B} B / 2$ breaks the valley symmetry of the levels, $g_{v}^{\prime}=4^{38,39}$. The eigenvalues $E_{\mu}^{s, \tau}(k)$ of Eq. (1), when the magnetic field is absent, are

$$
E_{\mu}^{s, \tau}(k)=\hbar v_{F} \varepsilon_{\mu}^{s, \tau}(k) .
$$

The subscript $\mu=\left(\mu_{1}, \mu_{2}\right)$ is used for labeling the energy bands: $\mu_{1}=+1(-1)$ is for the electron (hole) branches and $\mu_{2}=+1(-1)$ is for the upper (lower) layer. Using the label $\mu_{2}$ is allowed provided the interlayer coupling is weak, see Refs. 23 and 26. The factor $\varepsilon_{\mu}^{s, \tau}(k) \equiv \varepsilon$ in Eq. (2) is the solution of the quartic equation

$$
\begin{gathered}
{\left[\left(\varepsilon+\xi_{5}^{s \tau}\right)\left(\varepsilon-\xi_{6}^{s \tau}\right)-k^{2}\right]\left[\left(\varepsilon+\xi_{7}^{s \tau}\right)\left(\varepsilon-\xi_{8}^{s \tau}\right)-k^{2}\right]} \\
-\gamma^{\prime 2}\left(\varepsilon-\xi_{6}^{s \tau}\right)\left(\varepsilon-\xi_{8}^{s \tau}\right)=0
\end{gathered}
$$

where $k \equiv k_{y}$ is the wave vector, $\varepsilon=E / \hbar v_{F}, \xi_{5}^{s \tau}=$ $\xi_{1}^{s \tau} / \hbar v_{F}, \xi_{6}^{s \tau}=\xi_{2}^{s \tau} / \hbar v_{F}, \xi_{7}^{s \tau}=\xi_{3}^{s \tau} / \hbar v_{F}, \xi_{8}^{s \tau}=\xi_{4}^{s \tau} / \hbar v_{F}$, and $\gamma^{\prime}=\gamma / \hbar v_{F}$. In the limit $\xi_{i}^{s \tau} \rightarrow 0, i=5, . ., 8$, we obtain the energy dispersion for bilayer graphene $\mathrm{u}^{40}$.

In the upper panel of Fig. 1 we plot the energy dispersion of bilayer $\mathrm{WSe}_{2}$ for field $E_{z}=0$ at both valleys. We remark the following: (i) The splitting between the levels due to SOI is finite in the $\mathrm{CB}$ given by $2 \lambda_{c}$ at $k=0$ in contrast to bilayer $\mathrm{MoS}_{2} \underline{23,24,26,27}$. Its means that fourfold degeneracy of $\mathrm{CB}$ in $\mathrm{WSe}_{2}$ is partially lifted. So, the bands are two-two fold degenerate whereas it is four fold degenerate in bilayer $\mathrm{MoS}_{2}$ at $k=0$. But, the splitting due to interlayer hopping is negligible in the CB.(ii) The value of interlayer hopping between the two layers is finite in the $\mathrm{VB}^{23}, 24,26,27$. So, splitting of levels in the $\mathrm{VB}$ is a combined effect of interlayer hopping and SOI given by $2\left[\lambda_{v}^{2}+\gamma^{2}\right]^{1 / 2}$ at $k=0$. This relation indicates that the VB is still split for $\lambda_{v}=0$ or $\gamma=0$. Further, levels in VB are also two-two fold degenerate as seen upper panel of Fig. (1). (iii) The gap between conduction and valence band edges is given by $2 \Delta-\lambda_{c}-\left[\lambda_{v}^{2}+\gamma^{2}\right]^{1 / 2}$ for $k=0$.

For $E_{z} \neq 0$ we plot the energy spectrum in the lower panels of Fig. 1. We note the following: (i) The field $E_{z}$ modifies the SOI splitting. We note that two-fold spin degeneracy of all the bands in the $\mathrm{CB}$ and VB at each valley is completely lifted in contrast to bilayer $\mathrm{MoS}_{2}$. However, bands have two-fold valley degeneracy i. e. energies of spin up and spin down bands at $K$ and $K^{\prime}$ valleys are same and vise versa. (ii) An interlayer splitting is obtained in both the CB and VB. Analytically we
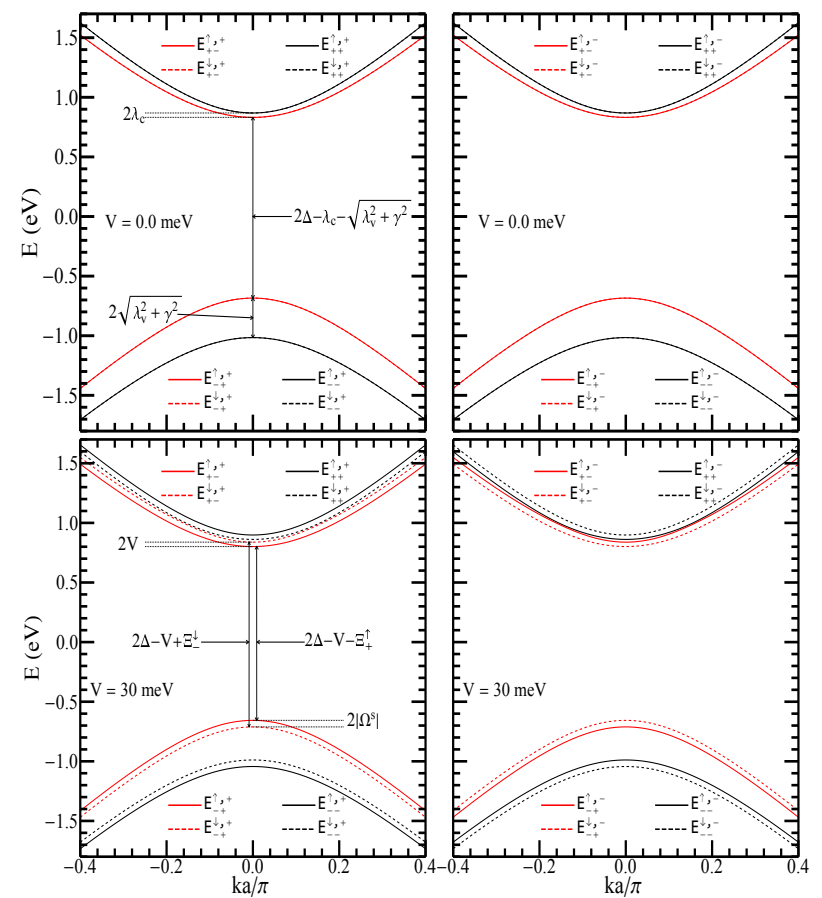

FIG. 1. Band structure of bilayer $\mathrm{WSe}_{2}$ for $2 \lambda_{c}=37 \mathrm{meV}$, $2 \lambda_{v}=303 \mathrm{meV}$, and $2 \gamma=134 \mathrm{meV}$. The upper panels are for $V=0 \mathrm{meV}$, the lower ones for $V=30 \mathrm{meV}$. The left (right) panels are for the $K\left(K^{\prime}\right)$ valley and $\Xi_{ \pm}^{s}=\lambda_{c} \pm \sqrt{\lambda_{v}^{2}+\gamma^{2}}+\Omega^{s}$ with $\Omega^{s}=s \lambda_{v} V /\left[\lambda_{v}^{2}+\gamma^{2}\right]^{1 / 2}$.

obtain the gaps $2 V \lambda_{v} /\left[\lambda_{v}^{2}+\gamma^{2}\right]^{1 / 2}$ for $V \ll \lambda_{v}$ and $2 V$ at the valence and conduction bands edges, respectively. (iii) The band gap is also reduced by the field $E_{z} \propto V$. It is equal to $2 \Delta-V-s \lambda_{c}-\left[\lambda_{v}^{2}+\gamma^{2}\right]^{1 / 2}-\tau s \lambda_{v} V /\left[\lambda_{v}^{2}+\gamma^{2}\right]^{1 / 2}$ for $V \ll \lambda_{v}$.

\section{A. Landau levels}

In the presence of a magnetic field $B$ perpendicular to the layers we replace $\pi$ by $-i \hbar \nabla+\mathbf{A}$ in Eq. (10) and take the vector potential $\mathbf{A}$ in the Landau gauge $\mathbf{A}=$ $(0, B x, 0)$. After diagonalizing Eq. (11) the Landau level (LL) spectrum is obtained as

$$
E_{n, \mu}^{s, \tau}=\hbar \omega_{c} \varepsilon_{n, \mu}^{s, \tau},
$$

with $\omega_{c}=v_{F} \sqrt{2 e B / \hbar}$ the cyclotron frequency. For $n \geq 1$ the factor $\varepsilon_{n, \mu}^{s, \tau} \equiv \varepsilon$ is the solution of the quartic equation

$$
\begin{gathered}
{\left[\left(\varepsilon+d_{1}^{s \tau}\right)\left(\varepsilon-d_{2}^{s \tau}\right)-n\right]\left[\left(\varepsilon+d_{3}^{s \tau}\right)\left(\varepsilon-d_{4}^{s \tau}\right)-(n+1)\right]} \\
-t^{2}\left(\varepsilon-d_{2}^{s \tau}\right)\left(\varepsilon-d_{4}^{s \tau}\right)=0,
\end{gathered}
$$

where $t=\gamma / \hbar \omega_{c}, d_{1}^{s \tau}=\kappa^{\tau}+s \lambda_{v}+\tau\left(s M_{z}-\tau M_{v}\right) / \hbar \omega_{c}$, $d_{2}^{s \tau}=\alpha^{\tau}-s \lambda_{c}-\tau\left(s M_{z}-\tau M_{v}\right) / \hbar \omega_{c}, d_{3}^{s \tau}=\alpha^{\tau}-s \lambda_{v}-$ $\tau\left(s M_{z}-\tau M_{v}\right) / \hbar \omega_{c}$, and $d_{4}^{s \tau}=\kappa^{\tau}+s \lambda_{c}+\tau\left(s M_{z}-\right.$ $\left.\tau M_{v}\right) / \hbar \omega_{c}$ with $\kappa^{\tau}=\Delta+\tau V$ and $\alpha^{\tau}=\Delta-\tau V$. In the limit $\xi_{i}^{s \tau} \rightarrow 0, i=5, . ., 8$, Eq. (4) gives a LL dispersion 
similar to that of bilayer graphene $\stackrel{41,42}{ }$. The eigenfunctions are

$$
\begin{gathered}
\psi_{n, \mu}^{s,+}=\frac{1}{\sqrt{L_{y}}}\left(\begin{array}{c}
\varrho_{n, \mu}^{s,+} \phi_{n} \\
\Theta_{n, \mu}^{s,+} \phi_{n-1} \\
\Lambda_{n, \mu}^{s,+} \phi_{n} \\
\Upsilon_{n, \mu}^{s,+} \phi_{n+1}
\end{array}\right) e^{i k_{y} y}, \\
\psi_{n, \mu}^{s,-}=\frac{1}{\sqrt{L_{y}}}\left(\begin{array}{c}
\Lambda_{n, \mu}^{s,-} \phi_{n} \\
\Upsilon_{n, \mu}^{s,-} \phi_{n+1} \\
\varrho_{n, \mu}^{s,-} \phi_{n} \\
\Theta_{n, \mu}^{s,-} \phi_{n-1}
\end{array}\right) e^{i k_{y} y} .
\end{gathered}
$$

Here $\phi_{n} \equiv \phi_{n}(v)=\left(2^{n} n ! l_{B} \sqrt{\pi}\right)^{-1 / 2} e^{-v^{2} / 2} H_{n}(v)$ is the harmonic oscillator wave function with $v=\left(x-l_{B}^{2} k_{y}\right) / l_{B}$ and $H_{n}(v)$ the Hermite polynomial of order $n$. Notice that $\phi_{n} \equiv 0$ for $n<0$. The coefficients are given by $\Theta_{n, \mu}^{s, \tau}=\sqrt{n} \varrho_{n, \mu}^{s, \tau} /\left[\varepsilon_{n, \mu}^{s, \tau}-d_{2}^{s \tau}\right], \Lambda_{n, \mu}^{s, \tau}=k_{n, \mu}^{s, \tau} \varrho_{n, \mu}^{s, \tau}$, and $\Upsilon_{n, \mu}^{s, \tau}=$ $\sqrt{n+1} k_{n, \mu}^{s, \tau} \varrho_{n, \mu}^{s, \tau} /\left[\varepsilon_{n, \mu}^{s, \tau}-d_{4}^{s \tau}\right]$, with $\varrho_{n, \mu}^{s, \tau}$ the normalization constants

$\varrho_{n, \mu}^{s, \tau}=\left\{\left(k_{n, \mu}^{s, \tau}\right)^{2}\left[1+\frac{(n+1)}{\left(\varepsilon_{n, \mu}^{s, \tau}-d_{4}^{s \tau}\right)^{2}}\right]+1+\frac{n}{\left(\varepsilon_{n, \mu}^{s, \tau}-d_{2}^{s \tau}\right)^{2}}\right\}^{-1}$

and $k_{n, \mu}^{s, \tau}=\left[\left(\varepsilon_{n, \mu}^{s, \tau}+d_{1}^{s \tau}\right)\left(\varepsilon_{n, \mu}^{s, \tau}-d_{2}^{s \tau}\right)-n\right] / t\left(\varepsilon_{n, \mu}^{s, \tau}-d_{2}^{s \tau}\right)$. As Eq. (6) shows, the full wave function is a mixture of the Landau wave functions with indices $n-1, n$, and $n+1$.

For $n=0$ there are two special LLs. One has the energies $\varepsilon_{0,+-}^{s,+}=d_{4}^{s+}$ and $\varepsilon_{0,+-}^{s,-}=d_{2}^{s-}$ for the $K$ and $K^{\prime}$ valleys, respectively. The corresponding wave functions are

$$
\psi_{0,+-}^{s,+}=\frac{1}{\sqrt{L_{y}}}\left(\begin{array}{c}
0 \\
0 \\
0 \\
\phi_{0}
\end{array}\right) e^{i k_{y} y}, \quad \psi_{0,+-}^{s,-}=\frac{1}{\sqrt{L_{y}}}\left(\begin{array}{c}
0 \\
\phi_{0} \\
0 \\
0
\end{array}\right) e^{i k_{y} y}
$$

This LL has exactly the same properties as the $n=0$ conventional, non-relativistic LL. For $\Delta=\lambda_{c}=\lambda_{v}=$ $V=0$, this level has exactly zero energy as the $n=0 \mathrm{LL}$ for bilayer graphene 41,42 . Also, from Eq. (5) we obtain three other levels for $n=0$. We obtain the wave functions for two of these levels from Eq. (6) by simply setting $n=0$ in it. Further, we specify the quantum number $(\mu)$ labels for these two levels as $\mu=(+,+)$ and $\mu=(-,+)$. However, for the third LL we specify $n$ and $\mu$ as shown in the eigenfunctions

$$
\begin{aligned}
& \psi_{0,--}^{s,+}=\frac{1}{\sqrt{L_{y}}}\left(\begin{array}{c}
\varrho_{0,--}^{s,+} \phi_{0} \\
0 \\
\Lambda_{0,--}^{s,+} \phi_{0} \\
\varrho_{0,--}^{s,+} t \phi_{1}
\end{array}\right) e^{i k_{y} y} \\
& \psi_{0,--}^{s,-}=\frac{1}{\sqrt{L_{y}}}\left(\begin{array}{c}
\Lambda_{0,--}^{s,-} \phi_{0} \\
\varrho_{0,--}^{s,-} t \phi_{1} \\
\varrho_{0,--}^{s,-} \phi_{0} \\
0
\end{array}\right) e^{i k_{y} y},
\end{aligned}
$$
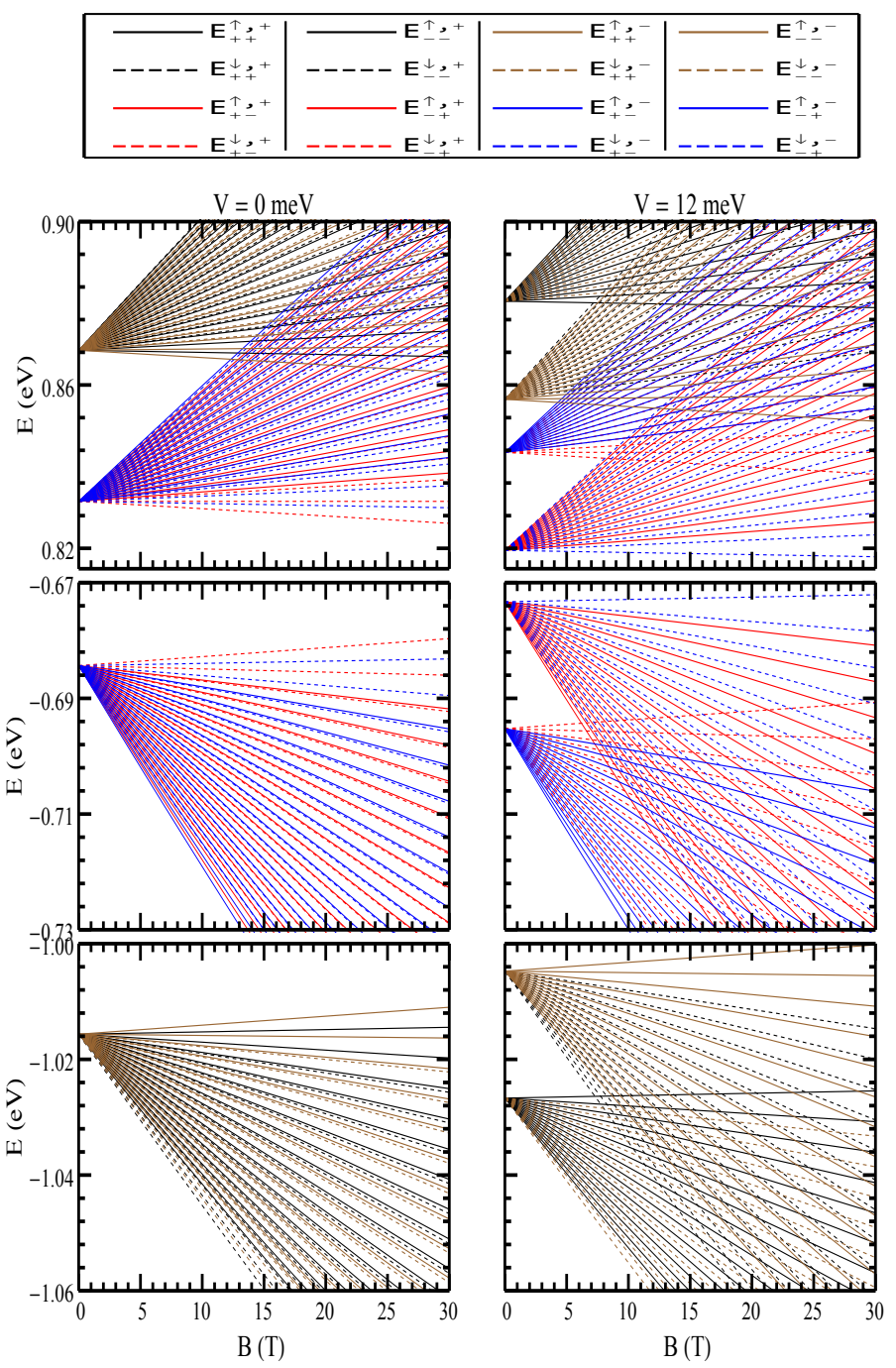

FIG. 2. Energy spectrum of bilayer $\mathrm{WSe}_{2}$ versus magnetic field $B$ for $M_{z} \neq, M_{v} \neq 0$. The left panel is for the $E_{z}=0$ and right one for $E_{z} \neq 0$, respectively. The upper panel explains the colour and style assignments of the curves.

where $\Lambda_{0,--}^{s, \tau}=\varrho_{0,--}^{s, \tau} t\left(\varepsilon_{0,--}^{s, \tau}-d_{4}^{s, \tau}\right)$. The normalization constants are

$$
\varrho_{0,--}^{s, \tau}=k_{0,--}^{s, \tau}\left\{\left(k_{0,--}^{s, \tau}\right)^{2}+t^{2}\left[1+\left(\varepsilon_{0,--}^{s, \tau}-d_{4,--}^{s, \tau}\right)\right]\right\}^{-1 / 2}
$$

and $k_{0,--}^{s, \tau}=\left(\varepsilon_{0,--}^{s, \tau}+d_{3,--}^{s, \tau}\right)\left(\varepsilon_{0,--}^{s, \tau}-d_{4,--}^{s, \tau}\right)-1$. The wave function corresponding to this LL is a mixture of the $n=0$ and $n=1$ conventional (nonrelativistic) Landau functions $\phi_{0}$ and $\phi_{1}$. For $\Delta=\lambda_{c}=\lambda_{v}=V=0$ Eq. (9) gives the eigenfunctions for bilayer graphene $\$ 1,42$.

In Fig. 2 (left panels) we plot the spin and valley dependent LL spectrum, but independent of $k_{y}$, given by Eq. (4) versus the magnetic field $B$ for $V=0$ and finite spin $M_{z}$ and valley $M_{v}$ Zeeman fields. The marking of all curves is explained in the upper panel. We find the following: (i) The energy spectrum grows linearly with $B$ due to the huge band gap. (ii) For $M_{z}=M_{v}=0$, all LLs $(n \geq 1)$ are two-fold degenerate corresponding 
to the two valleys including the $n=0 \mathrm{LL}$ with energies $\varepsilon_{0,++}^{s, \tau}, \varepsilon_{0,-+}^{\downarrow,+}, \varepsilon_{0,-+}^{\uparrow,-}$, and $\varepsilon_{0,--}^{\downarrow, \tau}$ in both the conduction and valence bands. The LL with energy $\varepsilon_{0,+-}^{s, \tau}=\Delta+s \lambda_{c}$ for $n=0$ is doubly degenerate in the conduction band, i.e. $\varepsilon_{0,+-}^{\uparrow,+} \equiv \varepsilon_{0,+-}^{\uparrow,-}$ and $\varepsilon_{0,+-}^{\downarrow,+} \equiv \varepsilon_{0,+-}^{\downarrow,-}$. Further, in the valence band the $n=0 \mathrm{LL}$ is two-fold valley degenerate, i.e. $\varepsilon_{0,-+}^{\uparrow,+} \equiv \varepsilon_{0,-+}^{\downarrow,-}$ and $\varepsilon_{0,--}^{\uparrow,+} \equiv \varepsilon_{0,--}^{\uparrow,-}$. In this situation, interlayer splitting among the levels of $\mathrm{WSe}_{2}$ or $\mathrm{MoS}_{2}$ bilayer is zero 27 . On the other hand, the intra-layer spin splitting in bilayer $\mathrm{WSe}_{2}$ is significantly large given by $2 \lambda_{c}$, which can be clearly seen in the limit of vanishing $B$ as compared to bilayer $\operatorname{MoS}_{2} 27$. (iii) For $M_{z} \neq 0, M_{v} \neq 0$, shown in the left panel of Fig. 2, the spin and valley degeneracies of all LLs $(n \geq 0)$ are lifted i.e., the energies of the spin-up (-down) LLs at the $K$ valley are different than the spin-down (-up) ones at the $K^{\prime}$ valley in contrast to the $B=0$ case. (iv) The valley Zeeman term $M_{v}$ lifts the spin degeneracy as well as the valley degeneracy in both the conduction and valence bands. This effect on the LLs, due to the $M_{v}$ term, is absent in bilayer $\mathrm{MoS}_{2} 27$. Notice that the inter-layer splitting among the levels of bilayer $\mathrm{WSe}_{2}$ vanishes in contrast to bilayer $\mathrm{MoS}_{2}{ }^{27}$.

We show the LL spectrum in Fig. 2 (right panels) for finite field $E_{z}(V=12 \mathrm{meV})$ including the $M_{z}$ and $M_{v}$ terms. We deduce the following: (i) The field $E_{z}$ modifies the interlayer splitting, e.g., it makes it $24 \mathrm{meV}$ and 23 $\mathrm{meV}$ in the conduction and valence bands, respectively. (ii) The spin and valley degeneracies of all levels $(n \geq 0)$ are completely lifted, i.e., the energies of the spin-up ( $\uparrow$ ) states at the $K$ valley and a spin-down $(\downarrow)$ ones at the $K^{\prime}$ valley are totally different in contrast to the $B=$ 0 case. Moreover, we can adjust the LL separation by varying the external electric and magnetic fields. This becomes important when we tune the onset frequency of the magneto-optical conductivity.

\section{B. Density of states}

The density of states $D(E)$ is given by

$$
D(E)=\frac{1}{S_{0}} \sum_{n, \tau, s, \mu, k_{y}} \delta\left(E-E_{n, \mu}^{s, \tau}\right)
$$

where $S_{0}=L_{x} L_{y}$ is the area of the system. The sum over $k_{y}$ can be calculated by using $k_{0}=L_{x} / 2 l_{B}^{2}$ and the prescription $\sum_{k_{y}} \rightarrow\left(L_{y} / 2 \pi\right) g_{s} g_{v} \int_{-k_{0}}^{k_{0}} d k_{y}=\left(S_{0} / D_{0}\right) g_{s} g_{v}$, with $D_{0}=2 \pi l_{B}^{2} ; g_{s}\left(g_{v}\right)$ denotes the spin (valley) degeneracy. In this work we take $g_{s}=g_{v}=1$ because the spin and valley degeneracies are lifted. $E_{F}$ at constant electron concentration $n_{e}$ we obtain $E_{F}$ from the relation

$$
n_{e}=\int_{-\infty}^{\infty} D(E) f(E) d E=\frac{g_{s / v}}{D_{0}} \sum_{n, \tau, s, \mu} f\left(E_{n, \mu}^{s, \tau}\right)
$$

where $f\left(E_{n, \mu}^{s, \tau}\right)=1 /\left[1+\exp \left[\beta\left(E_{n, \mu}^{s, \tau}-E_{F}\right)\right]\right]$ is the FermiDirac function and $\beta=1 / k_{B} T$.

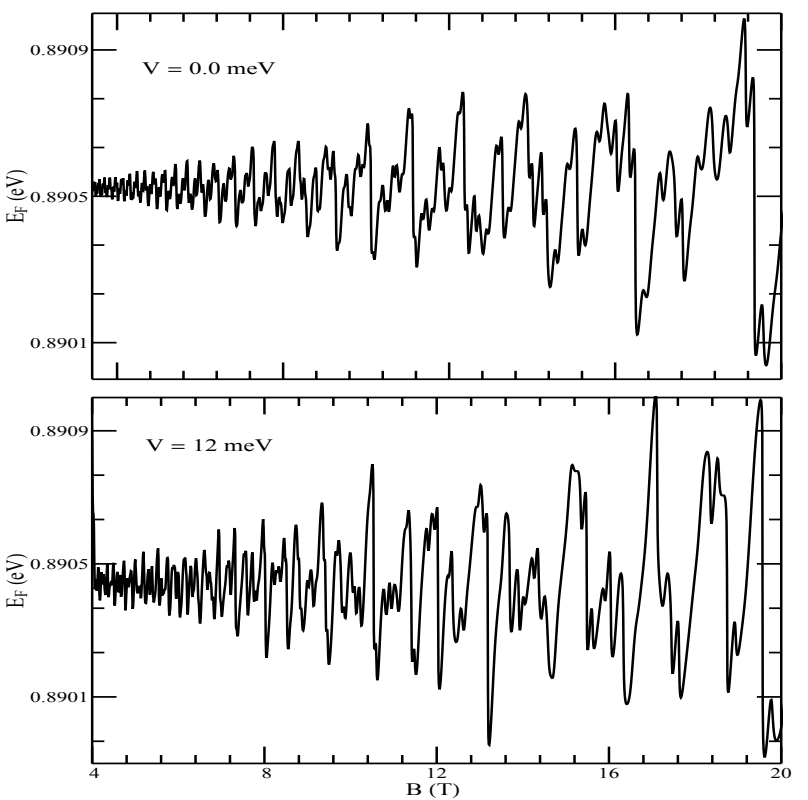

FIG. 3. Fermi energy $E_{F}$ versus $B$ for an electron density $n_{e}=4.3 \times 10^{13} \mathrm{~cm}^{-2}$. The upper panels are for $V=0 \mathrm{meV}$ and the lower ones for $V=12 \mathrm{meV}$.

The black solid curve in the upper panels of Fig. 3 shows $E_{F}$, obtained from Eq. (12) numerically, versus $B$ for $E_{z}=0$. The field $B$ lifts the spin and valley degeneracies of all LLs $(n \geq 0)$, i.e. the spin-up and spin-down electrons in the $K$ valley have different energies than the corresponding ones in the $K^{\prime}$ valley. This leads to additional intra-LL small jumps in Fig. 3 (upper panels) that are enhanced, as shown in the lower panels of Fig. 3 , when a finite electric field $E_{z}$ is applied.

We evaluate $D(E)$ per unit area assuming a Gaussian broadening of the $\delta$ function in Eq. (11). At zero temperature we have $D(E)=\left(g_{s} g_{v} / D_{0} \Gamma \sqrt{2 \pi}\right) \sum_{\zeta} \exp [-(E-$ $\left.\left.E_{\zeta}\right)^{2} / 2 \Gamma^{2}\right]$, where $\Gamma$ is the width of the distribution and $|\zeta\rangle \equiv\left|n, \mu, s, \tau, k_{y}\right\rangle$. In Fig. 4 we plot the dimensionless $D(E)$ versus the field $B$ in the conduction band for two different values of $E_{z}$ and $\Gamma$. The Shubnikov-de Haas (SdH) oscillations are clearly shown. The level broadening effect becomes significant for weak $B$ fields due to the small LL separation. On the other hand, this effect may become very weak in strong fields $B$ for which the LL separation is strong and $\Gamma \propto \sqrt{B}$.

Looking closely at Fig. 4 we observe a beating of the SdH oscillations at low fields $B$ and a pronounced splitting at higher fields. The beating of the oscillations is observed for $B \leq 10 \mathrm{~T}$, with $E_{z}=0$, and for $B \leq 5 \mathrm{~T}$ with $E_{z} \neq 0$. Away from these ranges the beating pattern is replaced by a split in the $\mathrm{SdH}$ oscillations. This behaviour is explained by the closeness of the oscillation frequencies of the SOI-split LLs. The field $B$ enhances the splitting in the conduction band by mixing the spinup and spin-down states of neighbouring LLs into two unequally spaced energy branches. This is also the case of a $2 \mathrm{DEG}^{43}$. This beating pattern occurs when the level 

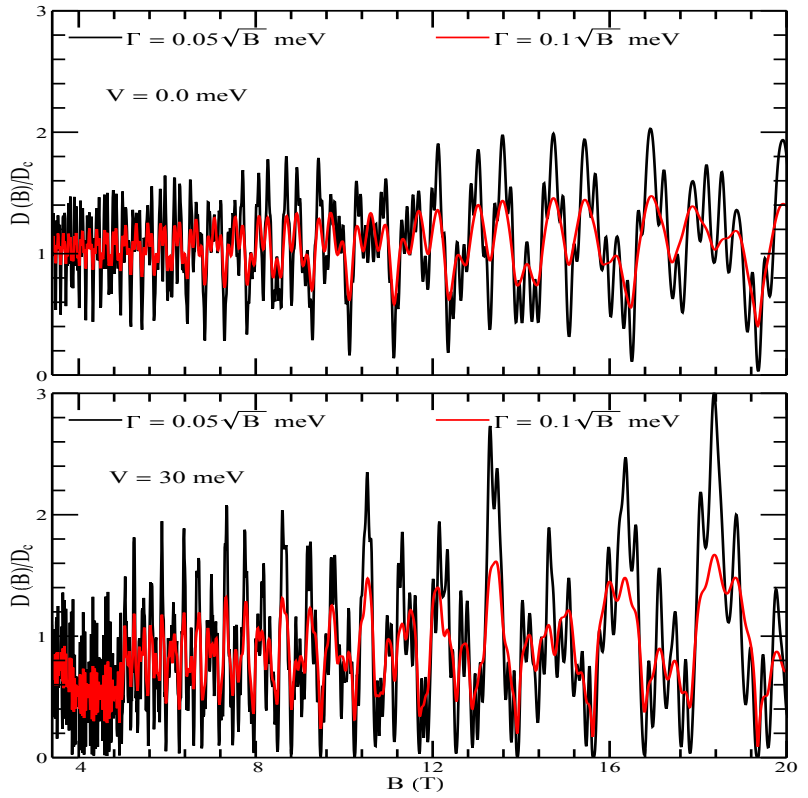

FIG. 4. Dimensionless density of states as a function of field $B$ for LL width $\Gamma=0.05 \sqrt{B} \mathrm{meV}$ (black curve) and $\Gamma=0.1 \sqrt{B}$ meV (red curve). The upper panels are for $V=0$ and the lower ones for $V=30 \mathrm{meV}$. The left and right panels differ only in the magnetic field range ( $x$ axis).

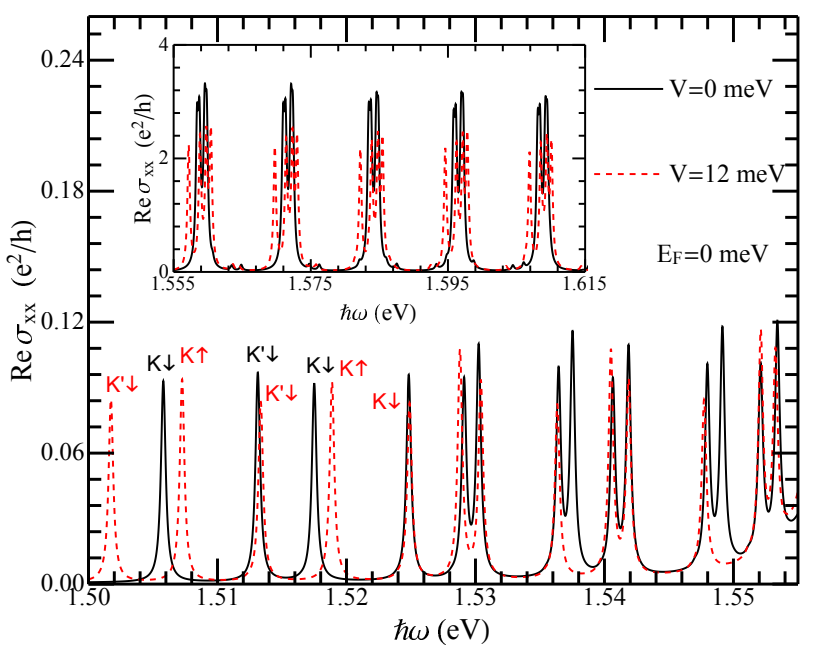

FIG. 5. Real part of the longitudinal optical conductivity $\sigma_{x x}^{n d}(\omega)$ versus the photon energy $\hbar \omega$ for a field $B=30 \mathrm{~T}$. The solid black and dotted red curves are for $V=0$ and $V=12$ meV, respectively. The inset shows $\operatorname{Re} \sigma_{x x}^{n d}(\omega)$ for higher $\hbar \omega$. The spin assignment of the curves follows from Eq. (18).

broadening is of the order of $\hbar \omega_{c}$; it is replaced by a split in the oscillations when the SOI becomes weak for large fields B. We further notice that the beating pattern shifts to lower magnetic fields for finite electric field energy $V$.

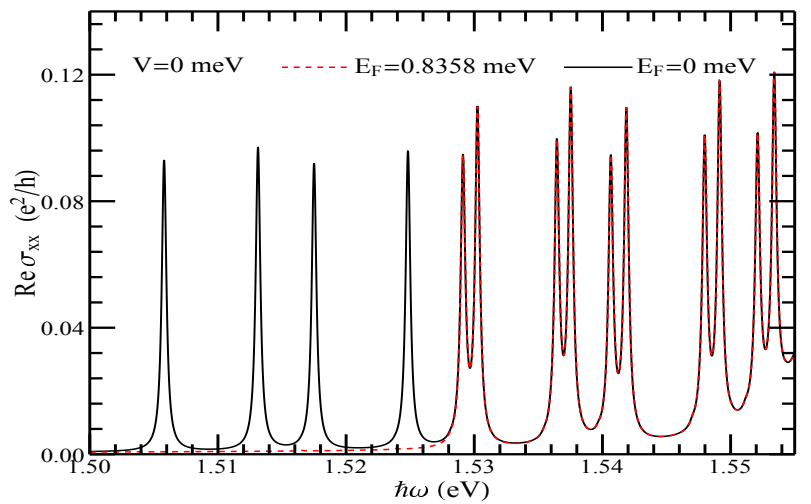

FIG. 6. As in Fig. 5 but for two different values of $E_{F}$ as indicated.

\section{CONDUCTIVITIES}

We consider a many-body system described by the Hamiltonian $H=H_{0}+H_{I}-\mathbf{R} \cdot \mathbf{F}(t)$, where $H_{0}$ is the unperturbed part, $H_{I}$ is a binary-type interaction (e.g., between electrons and impurities or phonons), and $-\mathbf{R} \cdot \mathbf{F}(t)$ is the interaction of the system with the external field $\mathrm{F}(\mathrm{t})^{\underline{44}}$. For conductivity problems we have $\mathbf{F}(t)=e \mathbf{E}(t)$, where $\mathbf{E}(t)$ is the electric field, $e$ the electron charge, $\mathbf{R}=\sum_{\mathbf{r}_{\mathbf{i}}}$, and $\mathbf{r}_{\mathbf{i}}$ the position operator of electron $i$. In the representation in which $H_{0}$ is diagonal the many-body density operator $\rho=\rho^{d}+\rho^{n d}$ has a diagonal part $\rho^{d}$ and a nondiagonal part $\rho^{n d}$. For weak electric fields and weak scattering potentials, for which the first Born approximation applies, the conductivity tensor has a diagonal part $\sigma_{\mu \nu}^{d}$ and a nondiagonal part $\sigma_{\mu \nu}^{n d}, \sigma_{\mu \nu}=\sigma_{\mu \nu}^{d}+\sigma_{\mu \nu}^{n d}, \mu, \nu=x, y$.

In general we have two kinds of currents, diffusive and hopping, with $\sigma_{\mu \nu}^{d}=\sigma_{\mu \nu}^{d i f}+\sigma_{\mu \nu}^{c o l}$, but usually only one of them is present. When a magnetic field is present we have only a hopping current since the diffusive part $\sigma_{\mu \nu}^{d i f}$ vanishes identically due to the vanishing velocity matrix elements as is evident, for elastic scattering, by its form 44

$$
\sigma_{\mu \nu}^{d}(\omega)=\frac{\beta e^{2}}{S_{0}} \sum_{\zeta} f_{\zeta}\left(1-f_{\zeta}\right) \frac{v_{\nu \zeta} v_{\mu \zeta} \tau_{\zeta}}{1+i \omega \tau_{\zeta}}
$$

where $\tau_{\zeta}$ is the momentum relaxation time, $\omega$ the frequency, and $v_{\mu \zeta}$ the diagonal matrix elements of the velocity operator. Further, $f_{\zeta}=\left[1+\exp \beta\left(E_{\zeta}-E_{F}\right)\right]^{-1}$ is the Fermi-Dirac distribution function, $\beta=1 / k_{B} T, T$ the temperature, and $S_{0}$ the area of the sample. In our case $v_{\mu \zeta}=0$ and the conductivity given by Eq. (13) vanishes.

The ac hopping conductivity $\sigma_{\mu \nu}^{c o l}(\omega)$ is given by Eq. (2.64) of Ref. [44]. In strong fields $B$ it is much smaller than the contribution $\sigma_{\mu \nu}^{n d}$, given below, and is neglected. Regarding this contribution $\sigma_{\mu \nu}^{n d}$ one can use the identity $f_{\zeta}\left(1-f_{\zeta^{\prime}}\right)\left[1-\exp \beta\left(E_{\zeta}-E_{\zeta^{\prime}}\right)\right]=f_{\zeta}-f_{\zeta^{\prime}}$ and cast the original form in the more familiar one $\underline{44}$ 

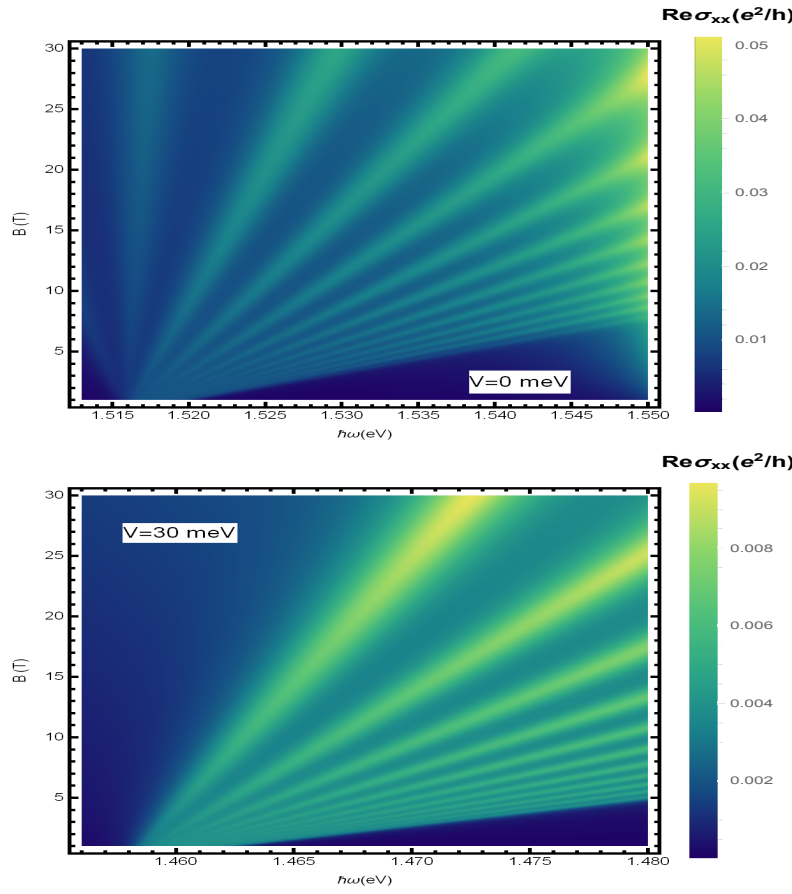

FIG. 7. $(B, \omega)$ Contour plot of the real part of the longitudinal conductivity for $E_{z}=0$ (upper panel) and $E_{z} \neq 0$ (lower panel). The level width $\Gamma$ is set to $0.4 \sqrt{B} \mathrm{meV}$.

$$
\sigma_{\mu \nu}^{n d}(\omega)=\frac{i \hbar e^{2}}{S_{0}} \sum_{\zeta \neq \zeta^{\prime}} \frac{\left(f_{\zeta}-f_{\zeta^{\prime}}\right) v_{\nu \zeta \zeta^{\prime}} v_{\mu \zeta \zeta^{\prime}}}{\left(E_{\zeta}-E_{\zeta^{\prime}}\right)\left(E_{\zeta}-E_{\zeta^{\prime}}+\hbar \omega-i \Gamma\right)}
$$

where the sum runs over all quantum numbers $|\zeta\rangle \equiv$ $\left|n, \mu, s, \tau, k_{y}\right\rangle$ and $\left|\zeta^{\prime}\right\rangle \equiv\left|n^{\prime}, \mu^{\prime}, s^{\prime}, \tau^{\prime}, k_{y}^{\prime}\right\rangle$ with $\zeta \neq \zeta^{\prime}$. The infinitesimal quantity $\epsilon$ in the original form $\frac{44}{4}$ has been replaced by $\Gamma_{\zeta} \approx \Gamma$ to account for the broadening of the energy levels. The familiar selection rules $n^{\prime}=n \pm 1$ are obtained through an evaluation of velocity matrix elements, see Eqs. (16)- (17) below. In the zerotemperature limit the Fermi function can be replaced by a step function. Further, we assume positive values of $E_{F}$, so that all transitions to negative levels are Pauli blocked. In Eq. (14) $v_{\nu \zeta \zeta^{\prime}}$ and $v_{\mu \zeta \zeta^{\prime}}$ are the off-diagonal matrix elements of the velocity operator. They are evaluated using the operator expressions $v_{x}=\partial H / \partial p_{x}$ and $v_{y}=\partial H / \partial p_{y}$, and are given in terms of the Pauli matrices $\sigma_{v}$ as

$$
v_{x}=\tau v_{F}\left(\begin{array}{cc}
\sigma_{x} & 0 \\
0 & \sigma_{x}
\end{array}\right), \quad v_{y}=v_{F}\left(\begin{array}{cc}
\sigma_{y} & 0 \\
0 & -\sigma_{y}
\end{array}\right)
$$

With $\varepsilon_{n, d_{2}} \equiv \varepsilon_{n, \mu}^{s, \tau}-d_{2}^{s \tau}, \varepsilon_{n, d_{4}} \equiv \varepsilon_{n, \mu}^{s, \tau}-d_{4}^{s \tau}$ and $Q=$ $v_{F} \varrho_{n, \mu}^{s, \tau} \varrho_{n^{\prime}, \mu^{\prime}}^{s^{\prime}, \tau^{\prime}} \delta_{s, s^{\prime}}$, and $R=k_{n, \mu}^{s, \tau} k_{n^{\prime}, \mu^{\prime}}^{s^{\prime}, \tau^{\prime}}$ the results are

$$
\begin{aligned}
& \left\langle\zeta\left|v_{x}\right| \zeta^{\prime}\right\rangle=\tau Q\left[\sqrt{n+1}\left(\frac{1}{\varepsilon_{n, d_{2}}^{\prime}}+\frac{R}{\varepsilon_{n, d_{4}}}\right) \delta_{n, n^{\prime}-1}+\sqrt{n}\left(\frac{1}{\varepsilon_{n, d_{2}}}+\frac{R}{\varepsilon_{n, d_{4}}^{\prime}}\right) \delta_{n, n^{\prime}+1}\right], \\
& \left\langle\zeta^{\prime}\left|v_{y}\right| \zeta\right\rangle=\tau i Q\left[\sqrt{n+1}\left(\frac{1}{\varepsilon_{n, d_{2}}^{\prime}}+\frac{R}{\varepsilon_{n, d_{4}}}\right) \delta_{n, n^{\prime}-1}-\sqrt{n}\left(\frac{1}{\varepsilon_{n, d_{2}}}+\frac{R}{\varepsilon_{n, d_{4}}^{\prime}}\right) \delta_{n, n^{\prime}+1}\right],
\end{aligned}
$$

where $\mu=\left\{\mu_{1}, \mu_{2}\right\}$. Using Eqs. (16), (17), and (14) we obtain the real and imaginary parts of the conductivities $\sigma_{x x}^{n d}(\omega)$ and $\sigma_{x y}^{n d}(\omega)$ which for convenience and later purposes we write, setting $\Delta_{n, n+1}=\varepsilon_{n, \mu}^{s, \tau}-\varepsilon_{n+1, \mu}^{s, \tau}$, as

$$
\begin{aligned}
\left(\begin{array}{c}
\operatorname{Re} \sigma_{x x}^{n d} \\
\operatorname{Im} \sigma_{x y}^{n d}
\end{array}\right) & =\mp \frac{e^{2}}{2 h} \sum_{s, \tau, n, \mu, \mu^{\prime}} \eta_{n, \mu, \mu^{\prime}}^{s, \tau} \bar{\Gamma}\left[\frac{1}{\left(\Delta_{n, n+1}+\bar{\omega}\right)^{2}+\bar{\Gamma}^{2}} \pm \frac{1}{\left(\Delta_{n, n+1}-\bar{\omega}\right)^{2}+\bar{\Gamma}^{2}}\right] \\
\left(\begin{array}{c}
\operatorname{Im} \sigma_{x x}^{n d} \\
\operatorname{Re} \sigma_{x y}^{n d}
\end{array}\right) & =-\frac{e^{2}}{2 h} \sum_{s, \tau, n, \mu, \mu^{\prime}} \eta_{n, \mu, \mu^{\prime}}^{s, \tau}\left[\frac{\Delta_{n, n+1}+\bar{\omega}}{\left(\Delta_{n, n+1}+\bar{\omega}\right)^{2}+\bar{\Gamma}^{2}} \mp \frac{\Delta_{n, n+1}-\bar{\omega}}{\left(\Delta_{n, n+1}-\bar{\omega}\right)^{2}+\bar{\Gamma}^{2}}\right]
\end{aligned}
$$

with

$$
\eta_{n, \mu, \mu^{\prime}}^{s, \tau}=(n+1)\left(\varrho_{n, \mu}^{s, \tau} \varrho_{n+1, \mu^{\prime}}^{s, \tau}\right)^{2}\left[\frac{k_{n, \mu}^{s, \tau} k_{n+1, \mu^{\prime}}^{s, \tau}}{\varepsilon_{n, d_{4}}}+\frac{1}{\varepsilon_{n+1, d_{2}}}\right]^{2} \frac{f\left(E_{n, \mu}^{s, \tau}\right)-f\left(E_{n+1, \mu^{\prime}}^{s, \tau}\right)}{\varepsilon_{n, \mu}^{s, \tau}-\varepsilon_{n+1, \mu^{\prime}}^{s, \tau}}
$$

Here $\bar{\omega} \equiv \omega / \omega_{c}$ and $\bar{\Gamma} \equiv \Gamma / \hbar \omega_{c}$. The Fermi Dirac func-

tion at $T=0$ becomes the Heaviside step function $\Theta(x)$ 


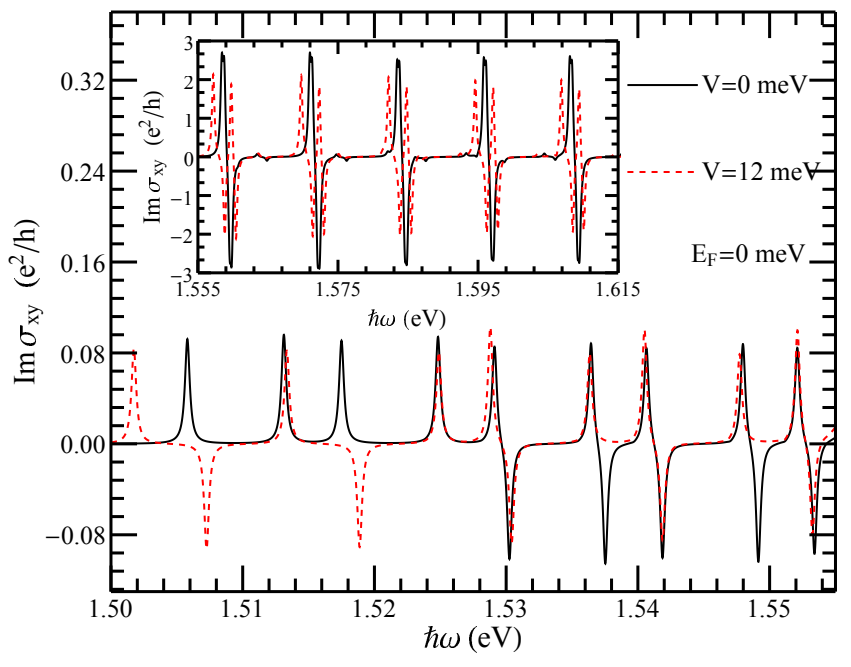

FIG. 8. As in Fig. 5 but for $\operatorname{Im} \sigma_{x y}$.

and enforces the Pauli exclusion principle for optical transitions, i.e., transitions occur only between the occupied $n$ state and the unoccupied $n^{\prime}$ one. The $n=0$ contributions to the absorptive conductivity Eq. (18) are evaluated separately. The results are given by Eq. (A4) in Appendix A.

Notice that in the limit $\omega \rightarrow 0, \Gamma \rightarrow 0$ we have

$$
\begin{aligned}
& \operatorname{Re} \sigma_{x x}^{n d}=\operatorname{Im} \sigma_{x x}^{n d}=\operatorname{Im} \sigma_{x y}^{n d}=0, \\
& \operatorname{Re} \sigma_{x y}^{n d}=-\frac{e^{2}}{h} \sum_{s, \tau, n, \mu, \mu^{\prime}} \frac{\eta_{n, \mu, \mu^{\prime}}^{s, \tau}}{\Delta_{n, n+1}} .
\end{aligned}
$$

The electron energies are different than those of the holes due to $\Delta$, the different values of the SOI and interlayer hopping (see Fig. 1). The terms intra-band and inter-band transitions refer to the bands in the absence of the magnetic field $(B=0)$. In bilayer $\mathrm{WSe}_{2}$ they belong to totally different regimes because of $\hbar \omega_{c}<<\Delta$ : the intra-band transitions fall in the microwave-to- $\mathrm{THz}$ regime and the inter-band ones in the visible frequency range because of the large value of the gap $\Delta$. Unlike bilayer graphene-like 2D systems, the asymmetry between the $\mathrm{CB}$ and $\mathrm{VB}$ in the bilayer $\mathrm{WSe}_{2}$ spectrum, due to the huge band gap and strong SOI, has important implications for the peaks seen in $\operatorname{Re} \sigma_{x x}^{n d}(\omega)$ and $\operatorname{Im} \sigma_{x y}^{n d}(\omega)$ as functions of the photon energy $(\hbar \omega)$.

The absorptive part of the longitudinal conductivity is shown in Fig. 5 for a temperature $T=0 \mathrm{~K}$ and a level broadening $\Gamma=0.04 \sqrt{B} \mathrm{meV}$. A larger magnetic field $(B=30 \mathrm{~T})$ has been used for well-resolved LL separation. The black solid and red dashed curves are for $E_{z}=$ 0 and $E_{z} \neq 0$, respectively. Here, we took $E_{F}=0 \mathrm{eV}$ in the gap. The optical selection rules allow $n$ to change by only 1, see Eqs. (16)-(17). In addition, one needs to go from occupied $(n)$ to unoccupied $\left(n^{\prime}\right)$ states through the absorption of photons with transitions allowed only between same-spin states. For $E_{z}=0$ and $E_{z} \neq 0$, the series of peaks occur at $\hbar \omega=-E_{n+1,-, \mu_{2}}^{s, \tau}+E_{n,+, \mu_{2}}^{s, \tau}$ and $\hbar \omega=-E_{n,-, \mu_{2}}^{s, \tau}+E_{n+1,+, \mu_{2}}^{s, \tau}$ for integer $n$. This series of peaks corresponds to the allowed inter-band transitions in the LL structure. As we can see from Fig. 5, the peaks are split due to the lifting of the spin and valley degeneracies in the presence of $B$ and absence of $E_{z}$ in contrast to the $B=0$ case. The spin-up transitions $-n \rightarrow(n+1)$ in $K\left(K^{\prime}\right)$ and spin-down ones $n \rightarrow-(n+1)$ in $K\left(K^{\prime}\right)$ are suppressed as seen by the small peaks in Fig. 5. On the other hand, the large peaks correspond to the spin-down transitions $-n \rightarrow(n+1)$ in $K\left(K^{\prime}\right)$ and the spin-up ones $n \rightarrow-(n+1)$ in $K\left(K^{\prime}\right)$.

When the electric field is applied, the splitting of the peaks increases and the peaks move to lower energies as well as to higher energies. Further, the spin and valley responses switch their labels. The shifting of peaks to lower energies signals the reduction of the band gap between CB and VB as can be seen in Figs. 1 and 2. Moreover, the shifting of the peaks to higher energies signals an increase of the gap between the $E_{n,++}^{s, \tau}\left(E_{n,--}^{s, \tau}\right)$ and $E_{n,+-}^{s, \tau}\left(E_{n,-+}^{s, \tau}\right)$ bands (see Figs. 1 and 2$)$. As the electric field is turned on, the intensity of the peaks is reduced due to a redistribution of the spectral weight between the peaks as shown by the red dotted curve in Fig. 5 . In contrast to monolayer $\mathrm{WSe}_{2} \frac{13}{}, \sigma_{x x}$ doesn't show any beating pattern at higher photon energies (not shown here) due to the well separated spin-up and spin-down states which do not mix at these frequencies. Another noteworthy point is that peak features in bilayer $\mathrm{WSe}_{2}$ are completely different than in bilayer graphene $\frac{45}{}$ due to the lack of perfect symmetry between the positive and negative branches (see Fig. 1).

A magnetic and electric control of the valley polarization can be clearly seen as the corresponding peaks in two different valleys appear at different frequencies. In addition to the valley-controlled transport, the peaks in each valley split as a result of all LLs becoming spin split. The spin and valley splittings can be understood with the help of Eq. (18) and the corresponding energies. One noteworthy feature, that becomes clear by comparing the black and red curves of Fig. 5, is that the peaks are well separated for $E_{z} \neq 0$ in both spin and valley spaces. In massless Dirac systems $\frac{46}{}$, the spin and valley peaks occur at the same frequency and hence a series of four peaks is replaced by one peak in contrast to bilayer $\mathrm{WSe}_{2}$ shown in Fig. 5. It is obvious from Fig. 5 that real absorptive part of $\sigma_{x x}$ of the bilayer WSe has a much richer structure than its monolayer counterpart 13 .

The effect of varying $E_{F}$ is shown in Fig. 6 for $E_{z}=0$. The value $E_{F}=0.8358 \mathrm{eV}$ is situated between the $n=0$ and $n=1 \mathrm{LLs}$, the first four peaks occurring at $\hbar \omega<1.53$ $\mathrm{eV}$ are completely removed due to Pauli blocking while all others $(\hbar \omega>1.53 \mathrm{eV})$ occur at the same energies as in Fig. 5. This behaviour is opposite to that of other 2D materials $\underline{46} \underline{\underline{49}}$ like graphene, silicene, $\alpha-T_{3}$ and topological insulators, in which the spectral weight of the interband peaks is continuously redistributed into the intra- 

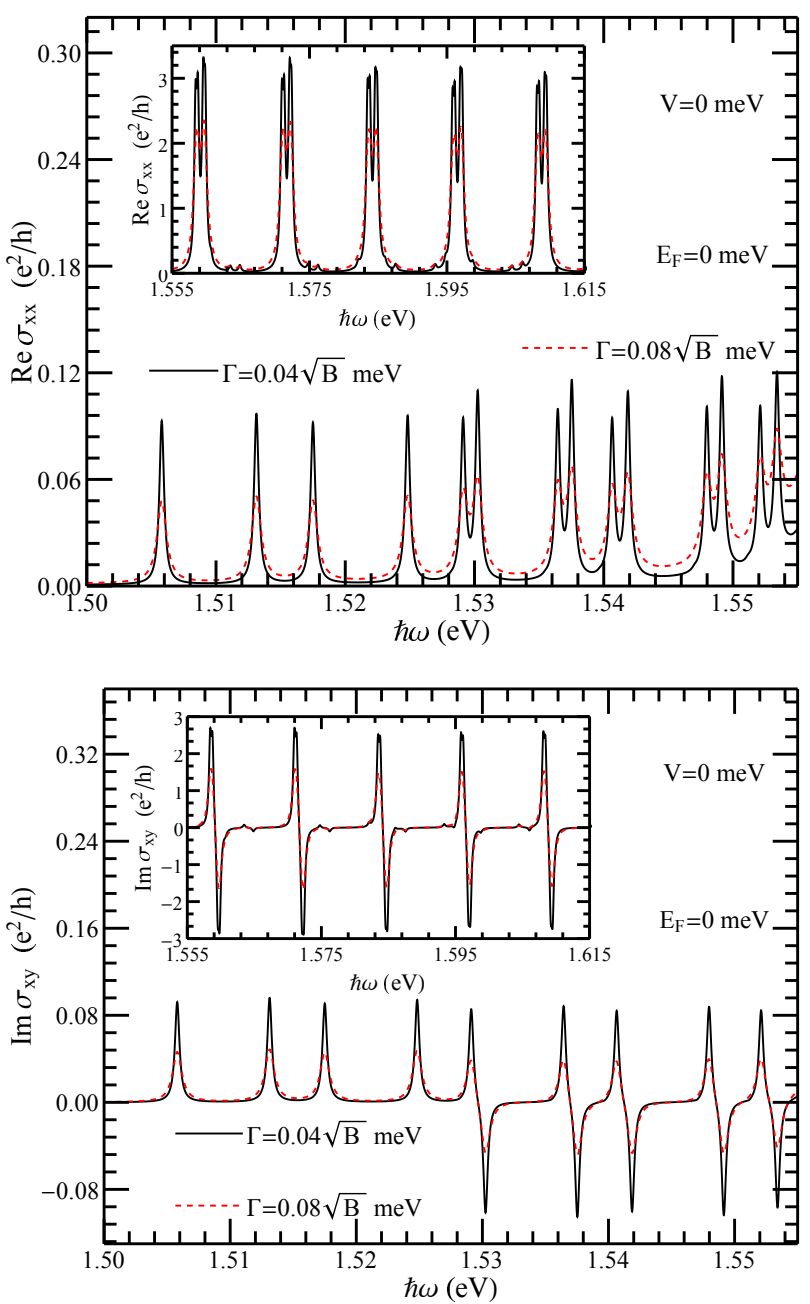

FIG. 9. The real part of $\sigma_{x x}$ (upper panel) and the maginary part of $\sigma_{x y}$ (lower panel) vs $\hbar \omega$ for $V=0, E_{F}=0 \mathrm{meV}$, $B=30 \mathrm{~T}$, and two values of the level width $\Gamma$.

band ones. This shows how the conductivity changes as $E_{F}$ moves through the LLs. Further, for $E_{z} \neq 0$ the lower peaks also disappear as $E_{F}$ moves to higher LLs.

For simplicity, we show a $(B, \omega)$ contour plot of $\operatorname{Re} \sigma_{x x}$ only for the $K$ valley in Fig. 7 aversus $B$ for two values of $V: V=0 \mathrm{meV}$ (upper panel) and $V=30 \mathrm{meV}$ (lower panel). In bilayer $\mathrm{WSe}_{2}$, as might be expected from Eq. (4), all observed transition energies behave linearly with the magnetic field $\left(\propto \hbar^{2} \omega_{c}^{2}\right)$. In contrast, in bilayer graphene 45 this occurs only for weak $B$ fields, but it switches over to a $\sqrt{B}$ dependence as the corresponding energy goes out of the parabolic band region. Also, the slope of the transition energies depends on the LL index $n$. In weak fields, the peaks are smeared out more easily in bilayer $\mathrm{WSe}_{2}$ than in its monolayer counterpart $\frac{13}{13}$. As expected, for $V \neq 0$, the peaks move to lower values of $\hbar \omega$ due to the reduction of the gap between the $\mathrm{CB}$ and VB (cf. lower panel of Fig. 7).

Figure 8 gives results for the $\operatorname{Im} \sigma_{x y}$ as a function of energy $\hbar \omega$ in $\mathrm{eV}$. The symmetry between positive and
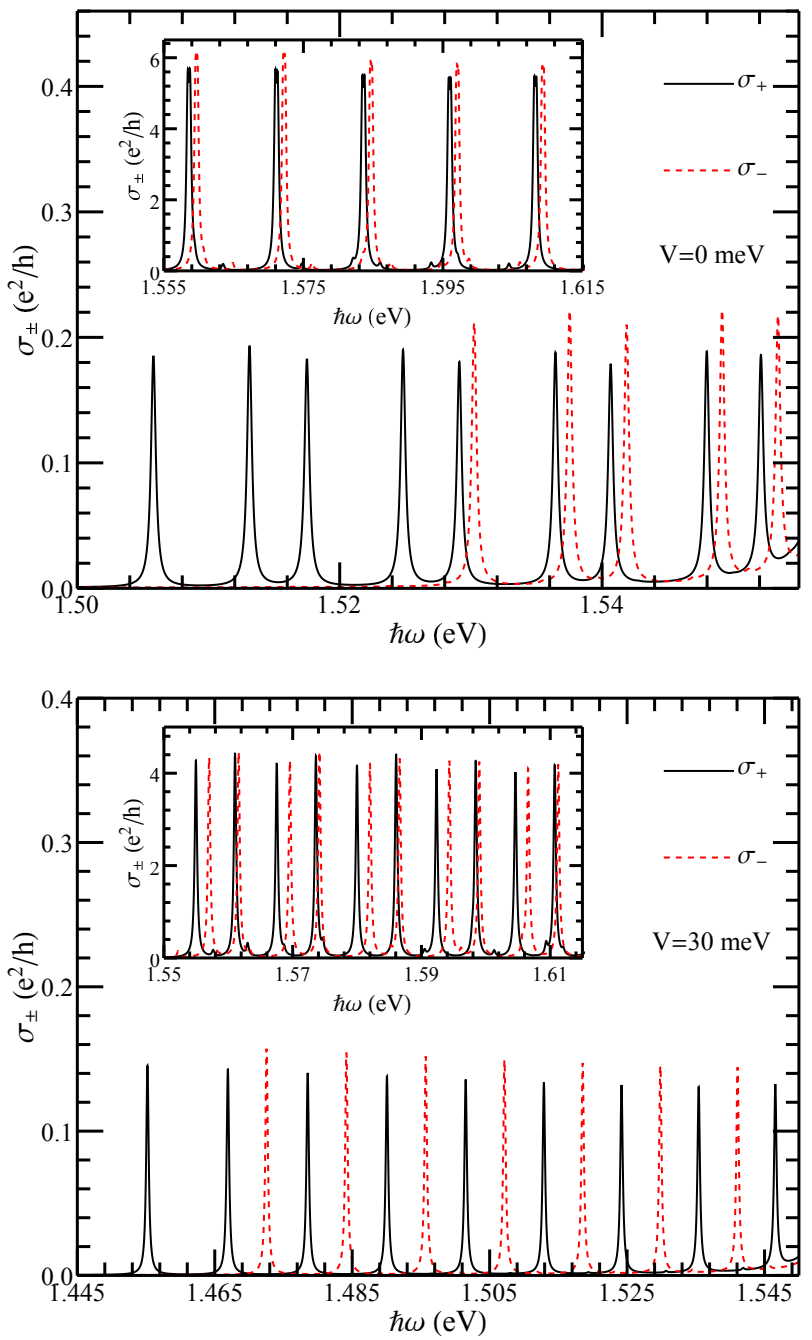

FIG. 10. Real part of the right polarized optical conductivity $\sigma_{+}(\omega)$ and of the left polarized one $\sigma_{-}(\omega)$ vs $\hbar \omega$ for $E_{F}=0$ meV and $B=30 \mathrm{~T}$. The solid black curve and red dotted one are for $V=0 \mathrm{meV}$ and $V=30 \mathrm{meV}$, respectively.

negative branches is no longer observed due to the $\Delta$ and SOI terms in Eq. (1), and the peaks corresponding to the transitions $-n \rightarrow(n+1)$ and $n \rightarrow-(n+1)$ have slightly different energies. Also, we can see the splitting of the conductivity peaks due to these transitions. The strength of the splitting directly reflects the energy difference between the $\mathrm{CB}$ and VB branches for the same $n$. The consequences of this difference is even more striking for the Hall conductivity than it is for the longitudinal one. So, we can see this mismatch as emergence of positive and negative oscillations in conductivity. This behaviour can also be understood by the negative sign between the two terms of Eq. (18). For the massless Dirac case, the negative and positive peaks would have the same energy and hence cancel out perfectly. Furthermore, there are no downward peaks in the range $\hbar \omega<1.53 \mathrm{eV}$ for $E_{z}=0$ but there are when the field $E_{z}$ is present.

In Fig. 9 we show the dependence of $\operatorname{Re} \sigma_{x x}$ and $\operatorname{Im} \sigma_{x y}$ 


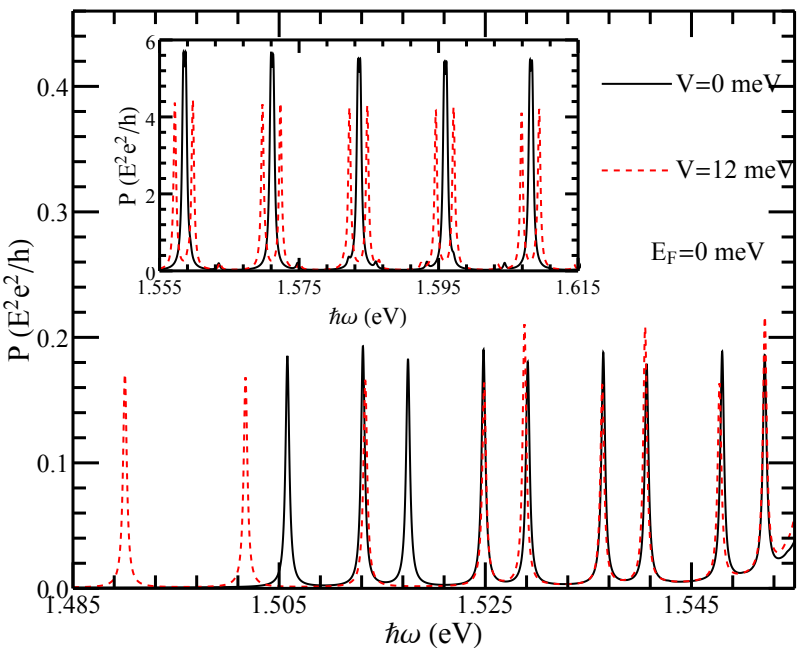

FIG. 11. Power spectrum vs $\hbar \omega$ for $V=0$ (black solid curve), $V=12 \mathrm{meV}$ (dotted red curve), and field $B=30 \mathrm{~T}$.

on the values of $\Gamma$. The solid black curve is for broadening $\Gamma=0.04 \sqrt{B} \mathrm{meV}$ and the red dotted one for $\Gamma=0.08 \sqrt{B}$ $\mathrm{meV}$. The separation of the split peaks becomes narrow with increasing broadening $\Gamma$. By further increasing $\Gamma$, the splitting of the peaks disappears because the broadening covers the spacing between the spin-split LLs. To retain these peaks one has to apply a magnetic field for which the spin splitting exceeds the LL broadening $\Gamma \propto \sqrt{B}$. In other words, a large $\Gamma$ smears out the peaks.

The peak structure just described above for $\operatorname{Re} \sigma_{x x}^{n d}(\omega)$ and $\operatorname{Im} \sigma_{x y}^{n d}(\omega)$ importantly affects the behaviour of the conductivity for right $(+)$ and left $(-)$ polarized light. For real experiments that probe the circular polarization of resonant light, as in the case of the Kerr and Faraday effects, one evaluates the quantity $\sigma_{ \pm}(\omega)$ given by

$$
\sigma_{ \pm}(\omega)=\operatorname{Re} \sigma_{x x}^{n d}(\omega) \pm \operatorname{Im} \sigma_{x y}^{n d}(\omega)
$$

with the $+(-)$ sign corresponding to the right (left) polarization. In Fig. 10 we show $\sigma_{-}(\omega)$ (dotted red curve) and $\sigma_{+}(\omega)$ (solid black curve) as functions of the frequency, for $E_{F}=0.0 \mathrm{eV}$ in the gap, with $E_{z}=0$ (upper panel) and $E_{z} \neq 0$ (lower panel), using the parameters of Fig. 5. As seen, there is a direct correspondence between these results and those of Figs. 5 and 8 . The heights of the peaks for $E_{z}=0$ and $E_{z} \neq 0$ in $\sigma_{-}(\omega)$ are slightly higher than those in $\sigma_{+}(\omega)$. Also, note that there is a double split-peak structure rather than a four split-peak structure as in $\sigma_{x x}(\omega)$. The peaks of $\sigma_{-}(\omega)$ and $\sigma_{+}(\omega)$ are displaced in energy with respect to each other. Similar to the behaviour of $\operatorname{Re} \sigma_{x x}^{n d}(\omega)$ and $\operatorname{Im} \sigma_{x y}^{n d}(\omega)$, the spin and valley splittings increase with $E_{z}$.

The difference between $\sigma_{-}(\omega)$ and $\sigma_{+}(\omega)$ is also reflected in the power absorption spectrum given by

$$
P(\omega)=(E / 2)\left[\sigma_{x x}(\omega)+\sigma_{y y}(\omega) i \sigma_{y x}(\omega)+i \sigma_{x y}(\omega)\right] .
$$

We recall that $\sigma_{\mu \nu}=\sigma_{\mu \nu}^{d}+\sigma_{\mu \nu}^{n d}=\sigma_{\mu \nu}^{n d}$ since the component $\sigma_{\mu \mu}^{d}, \mu=x, y$, vanishes. The component $\sigma_{y y}^{n d}(\omega)$ is
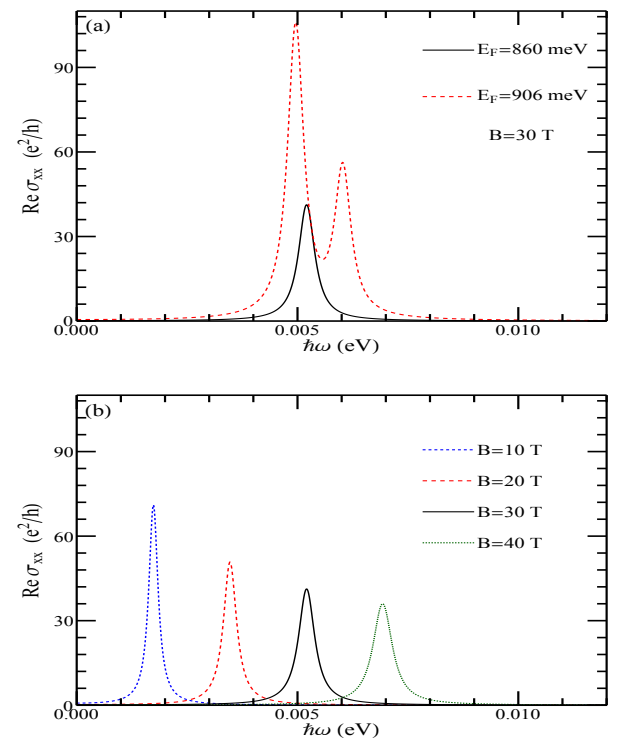

FIG. 12. (a) Intra-band limit of the real part of the longitudinal conductivity versus photon energy $\hbar \omega$ for $B=30 \mathrm{~T}$ and two values of $E_{F}$. (b) As in (a) for four values of $B$ and $E_{F}$ close to $860 \mathrm{meV}$ for $B \neq 30 \mathrm{~T}$. The energy $\hbar \omega$ is measured from the bottom of the conduction band.

given by $\sigma_{x x}^{n d}(\omega)$ and $\operatorname{Im} \sigma_{x y}^{n d}(\omega)=\operatorname{Im} \sigma_{y x}^{n d}(\omega)$. The spectrum $P(\omega)$ is shown in Fig. 11 as a function of the photon frequency for $E_{z}=0$ and $E_{z} \neq 0$. Given that $\operatorname{Im} \sigma_{x y}^{n d}(\omega)$ is the negative of $\operatorname{Re} \sigma_{x x}^{n d}(\omega)$, see Eq. (18), the peaks in it are essentially the same as those in the longitudinal optical conductivity but positive and negative. Similar to $\operatorname{Re} \sigma_{x x}^{n d}(\omega)$ and $\operatorname{Im} \sigma_{x y}^{n d}(\omega)$, spin and valley splittings can be clearly seen in Fig. 11 and for $E_{z} \neq 0$ the separation between them increases.

The semiclassical limit of the magneto-optical conductivity occurs when the magnetic field is very weak and the spacing becomes inconsequential. This occurs for a large Fermi energy, $E_{F} \gg \varepsilon_{0,+-}^{s, \tau}$. For $E_{F}>0$, only intra-band transitions are obtained between the $n$th and $(n+1)$ th LLs in the CB. For $n \gg 0$, consider $E_{F} \approx E_{n,+, \mu_{2}}$ lies between the $n$th and $(n+1)$ th LLs. In this limit, the energy spacing is linear in $B$ in contrast to the $\sqrt{B}$ behaviour in Weyl semimetals 50 . The pertinent energy difference is $E_{n,+, \mu_{2}}-E_{n+1,+, \mu_{2}}=-\hbar \omega_{c}$.

We show the results of $\operatorname{Re} \sigma_{x x}^{n d}(\omega)$ for the intra-band case in Fig. 12. We see from the upper panel that there is a spectral weight redistribution to a strong intraband response when $E_{F}$ increased. Furthermore, the optical spectral weight under these curves increases with $E_{F}$ in contrast to topological insulators $\underline{46}$. Further, a double peak response is present in the strong intra-band response as the dashed red curve in the upper panel shows. This results from the spin splitting of the LLs that renders the spin levels at a given valley unequal in energy. Also, the separation between the double peaks (red dashed curve) increases with $E_{F}$. Similar to the monolayer $\mathrm{WSe}_{2} \frac{13}{}$, these peaks lie in the range of microwave- 
to- $\mathrm{THz}$ frequencies and their height is larger than that of the inter-band transitions shown in Figs. 5-11. Further, when we increase the magnetic field $B$, as seen in the lower panel of Fig. 12, the intra-band peaks move to higher energies and their height is reduced in contrast to massless Dirac materials $\underline{46.47}$. For large $E_{F}$ the effect of $E_{z}$ becomes inconsequential. These results are consistent with graphene-like 2D systems in which the relevant spectral weight increases with $E_{F}$, while the optical features in these $2 \mathrm{D}$ systems lie only in the $\mathrm{THz}$ regime $\underline{\underline{46}} \underline{\underline{50}}$.

\section{SUMMARY AND CONCLUSIONS}

We have shown how the gap $\Delta$ and the SOI strength modify the electronic energy dispersion in bilayer $\mathrm{WSe}_{2}$, unlike bilayer graphene ${ }^{41.42}$, in the absence and presence of magnetic and electric fields. For $B=E_{z}=0$ and $B \neq E_{z} \neq 0$, the energies of the levels in the conduction and valence bands no longer mirror each other, cf. Figs. 1, 2. Further, we have studied the spin- and valleycontrolled magnetotransport in the presence and absence of $E_{z}$. We point out that inter-band optical transitions from level $n$ in the valence band to level $n+1$ in the conduction band no longer have the same energy as those from level $n+1$ to level $n$; this splits the corresponding absorption line in the real part of the longitudinal conductivity. Also, the optical spectral weight of these lines is different (see the large and small peaks of Fig. 4) from that in graphene. The energy of the splitting is related to the mismatch in energy levels between the conduction and valence bands, see Fig. 2. A similar splitting was found for the imaginary part of the Hall conductivity.

Due to the large $\Delta, \lambda_{c}$ and $\lambda_{v}$ terms, the conductiv- ity peaks in $\mathrm{WSe}_{2}$ depend linearly on $B$, contrary to bilayer graphene ${ }^{45}$, and reflect the equidistant LLs in each band. In addition, the onset energies of the spin- and valley-dependent transitions reflect the energy difference between the LLs and are controlled by the magnetic and electric fields. The other determining factors are the band gap and the SOI strength. Accordingly, we may expect that a careful tuning of electric and magnetic fields will determine the value of band gap and SOI strength. However, for the absorption of circularly polarized light, two-peak structures are recovered but in this case there is a shift in the energy position and amplitude of the lines between right and left polarizations in contrast to what is found when the band gap and SOI terms in the electron dispersion curves are zero for graphene.

The semiclassical limit is affected by the magnetic field. This significantly shifts not only the intra-band peak to higher $\hbar \omega$ values, but also reduces the peak amplitude in contrast with graphene. The lineshape associated with the intra-band magneto-conductivity is significantly changed when the Fermi energy is varied. The optical spectral weight under these curves is found to increase in contrast to topological insulators and similar massless Dirac systems $\underline{46}$. These novel findings may be pertinent to the development of spintronic and valleytronic optical devices based on bilayer TMDCs.

\section{ACKNOWLEDGMENTS}

M. Z. and P. V. acknowledge the support of the Canadian NSERC Grant No. OGP0121756. The work of M. T. was supported by Colorado State University.

\section{Appendix A: Zero-level Hall conductivity}

Using Eq. (6), and Eq. (8) the off-diagonal velocity matrix elements for $n=0$ are

$$
\begin{gathered}
\left\langle 0, \mu, s, \tau\left|v_{x}\right| n^{\prime}, \mu^{\prime}, s^{\prime}, \tau^{\prime}\right\rangle=\tau v_{F} \varrho_{0, \mu}^{s, \tau} \varrho_{n^{\prime}, \mu^{\prime}}^{s^{\prime}, \tau_{s, s^{\prime}}^{\prime}} \times\left\{\sqrt{n^{\prime}} / \varepsilon_{n, d_{2}^{\prime}}+k_{0, \mu}^{s, \tau} k_{n^{\prime}, \mu^{\prime}}^{s^{\prime}, \tau^{\prime}} / \varepsilon_{0, d_{4}}\right\} \delta_{0, n^{\prime}-1} \\
\left\langle n^{\prime}, \mu^{\prime}, s^{\prime}, \tau^{\prime}\left|v_{y}\right| 0, \mu, s, \tau\right\rangle=\tau i v_{F} \varrho_{0, \mu}^{s, \tau} \varrho_{n^{\prime}, \mu^{\prime}}^{s^{\prime}, \tau^{\prime}} \delta_{s, s^{\prime}} \times\left\{\sqrt{n^{\prime}} / \varepsilon_{n, d_{2}^{\prime}}+k_{0, \mu}^{s, \tau} k_{n^{\prime}, \mu^{\prime}}^{s^{\prime}, \tau^{\prime}} / \varepsilon_{0, d_{4}}\right\} \delta_{0, n^{\prime}-1} \\
\left\langle 0,+-, \tau\left|v_{x}\right| n^{\prime}, \mu^{\prime}, s^{\prime}, \tau^{\prime}\right\rangle=\tau v_{F} Y, \quad\left\langle n^{\prime}, \mu^{\prime}, s^{\prime}, \tau^{\prime}\left|v_{y}\right| 0,+-, \tau\right\rangle=\tau i v_{F} Y, \quad Y=\varrho_{n^{\prime}, \mu^{\prime}}^{s^{\prime}, \tau_{n^{\prime}, \mu^{\prime}}^{s^{\prime}, \tau^{\prime}} \delta_{s, s^{\prime}} \delta_{0, n^{\prime}},}
\end{gathered}
$$

Using these expressions the conductivities take the form

$$
\begin{aligned}
\left(\begin{array}{c}
\operatorname{Re} \sigma_{x x}^{n d} \\
\operatorname{Im} \sigma_{x y}^{n d}
\end{array}\right) & =\mp \frac{e^{2}}{2 h} \sum_{s, \tau, \mu, \mu^{\prime}} \eta_{0,1, \mu, \mu^{\prime}}^{s, \tau}\left[\frac{1}{\left(\varepsilon_{0, \mu}^{s, \tau}-\varepsilon_{1, \mu}^{s, \tau}+\bar{\omega}\right)^{2}+\bar{\Gamma}^{2}} \pm \frac{1}{\left(\varepsilon_{0, \mu}^{s, \tau}-\varepsilon_{1, \mu}^{s, \tau}-\bar{\omega}\right)^{2}+\bar{\Gamma}^{2}}\right] \\
& =\mp \frac{e^{2}}{2 h} \sum_{s, \tau, \mu^{\prime}} v_{0,+-, \mu^{\prime}}^{s, \tau}\left[\frac{1}{\left(\varepsilon_{0,+-}^{s, \tau}-\varepsilon_{0, \mu}^{s, \tau}+\bar{\omega}\right)^{2}+\bar{\Gamma}^{2}} \pm \frac{1}{\left(\varepsilon_{0,+-}^{s, \tau}-\varepsilon_{0, \mu}^{s, \tau}-\bar{\omega}\right)^{2}+\bar{\Gamma}^{2}}\right]
\end{aligned}
$$


where

$$
\eta_{0,1, \mu, \mu^{\prime}}^{s, \tau}=\bar{\Gamma}\left(\varrho_{0, \mu}^{s, \tau} \varrho_{1, \mu^{\prime}}^{s, \tau}\right)^{2}\left[\frac{1}{\varepsilon_{1, d_{2}}^{\prime}}+\frac{k_{0, \mu}^{s, \tau} k_{1, \mu^{\prime}}^{s, \tau}}{\varepsilon_{0, d_{4}}}\right]^{2} \frac{f_{0, \mu}^{s, \tau}-f_{1, \mu^{\prime}}^{s, \tau}}{\varepsilon_{0, \mu}^{s, \tau}-\varepsilon_{1, \mu^{\prime}}^{s, \tau}}, \quad v_{0,+-, \mu^{\prime}}^{s, \tau}=\bar{\Gamma}\left(\varrho_{0, \mu^{\prime}}^{s, \tau} k_{0, \mu^{\prime}}^{s, \tau}\right)^{2} \frac{f_{0,+-}^{s, \tau}-f_{0, \mu^{\prime}}^{s, \tau}}{\varepsilon_{+-}^{s, \tau}-\varepsilon_{0, \mu^{\prime}}^{s, \tau}}
$$

1 I. Zutic, J. Fabian, and S. Das Sarma, Rev. Mod. Phys. 76, 323 (2004).

2 T. Cao, G. Wang, W. Han, H. Ye, C. Zhu, J. Shi, Q. Niu, P. Tan, E. Wang, B. Liu, and J. Feng, Nat. Commun. 3, 887 (2012).

${ }^{3}$ K. F. Mak, C. Lee, J. Hone, J. Shan, and T. F. Heinz, Phys. Rev. Lett. 105, 136805 (2010).

4 B. Radisavljevic, A. Radenovic, J. Brivio, V. Giacometti, and A. Kis, Nature Nanotech. 6, 147 (2011).

5 A. Splendiani, L. Sun, Y. B. Zhang, T. S. Li, J. Kim, C. Y. Chim, G. Galli, and F. Wang, Nano Lett. 10, 1271 (2010).

6 A. Kuc, N. Zibouche, and T. Heine, Phys. Rev. B 83, 245213 (2011).

7 Z. Y. Zhu, Y. C. Cheng, and U. Schwingenschlögl, Phys. Rev. B 84, 153402 (2011).

8 L. Sun, J. Yan, D. Zhan, L. Liu, H. Hu, H. Li, B. K. Tay, J.-L. Kuo, C.-C. Huang, D. W. Hewak, P. S. Lee, and Z. X. Shen, Phys. Rev. Lett. 111, 126801 (2013).

9 H. Wang, L. Yu, Y.-H. Lee, Y. Shi, A. Hsu, M. L. Chin, L.-J. Li, M. Dubey, J. G. Kong, and T. Palacios, Nano Lett. 12, 4674 (2012).

10 O. Lopez-Sanchez, D. Lembke, M. Kayci, A. Radenovic, and A. Kis, Nat. Nanotechnol. 8, 497 (2013).

11 B. Radisavljevic, M. B. Whitwick, and A. Kis, Appl. Phys. Lett. 101, 043103 (2012).

12 X. Zhou, Y. Liu, M. Zhou, H. H. Shao, and G. H. Zhou, Appl. Phys. Express 7, 021201 (2014).

13 M. Tahir and P. Vasilopoulos, Phys. Rev. B 94, 045415 (2016).

14 X. Zhou, Y. Liu, M. Zhou, D. Tang, and G. Zhou, J. Phys.: Condens. Matter 26, 485008 (2014).

15 M. Tahir, P. Vasilopoulos, and F. M. Peeters, Phys. Rev. B 93, 035406 (2016).

16 A. Kormányos, P. Rakyta, and G. Burkard, New J. Phys. 17, 103006 (2015).

17 X. Cui, G.-H. Lee, Y. D. Kim, G. Arefe, P. Y. Huang, C.-H. Lee, D. A. Chenet, X. Zhang, L. Wang, F. Ye, F. Pizzocchero, B. S. Jessen, K. Watanabe, T. Taniguchi, D. A. Muller, T. Low, P. Kim, and J. Hone, Nat. Nanotechnol. 10, 534 (2015).

18 J. A. Wilson and A. D. Yoffe, Adv. Phys. 18, 193 (1969).

19 A. Enyashin, S. Gemming, and G. Seifert, Eur. Phys. J. Spec. Top. 149, 103 (2007).

20 M. Bar-Sadan, I. Kaplan-Ashiri, and R. Tenne, Eur. Phys. J. Spec. Top. 149, 71 (2007).

${ }^{21}$ R. Tenne and C. N. R. Rao, Philos. Trans. R. Soc. London A 362, 2099 (2004).

22 A. T. Neal, H. Liu, J. J. Gu, and P. D. Ye, ACS Nano 7, 7077 (2013).

23 Z. Gong, G.-B. Liu, H. Yu, D. Xiao, X. Cui, X. Xu, and W. Yao, Nat. Commun. 4, 15 (2013).

${ }^{24}$ S. Wu, J. S. Ross, G. B. Liu, G. Aivazian, A. Jones, Z. Fei, W. Zhu, D. Xiao, W. Yao, D. Cobden, and X. Xu, Nat.
Phys. 9, 149 (2013).

25 J. Lee, K. F. Mak, and J. Shan, Nat. Nanotechnol. 11, 421 (2016).

26 A. M. Jones, H. Yu, J. S. Ross, P. Klement, N. J. Ghimire, J. Yan, D. G. Mandrus, W. Yao, and X. Xu, Nat. Phys. 10, 130 (2014).

27 M. Zuabir, M. Tahir, P. Vasilopoulos, and K. Sabeeh, Phys. Rev. B 96, 045405 (2017).

28 Nourdine Zibouche, Pier Philipsen, Agnieszka Kuc, and Thomas Heine, Phys. Rev. B 90, 125440 (2014).

29 A. Ramasubramaniam, D. Naveh and E. Towe, Phys. Rev. B 84, 205325 (2011).

30 N. Ubrig, S. Jo, M. Philippi, D. Costanzo, H. Berger, A. B. Kuzmenko, and A. F. Morpurgo, Nano Letters 17 (9), 5719 (2017).

31 R. Suzuki, M. Sakano, Y. J. Zhang, R. Akashi, D. Morikawa, A. Harasawa, K. Yaji, K. Kuroda, K. Miyamoto, T. Okuda, K. Ishizaka, R. Arita, and Y. Iwasa, Nature Nano 9, 611 (2014).

32 R. Akashi, M. Ochi, S. Bordács, R. Suzuki, Y. Tokura, Y. Iwasa, and R. Arita, Phys. Rev. Applied 4, 014002 (2015).

33 J. Yan, J. Xia, X. Wang, L. Liu, J.-L. Kuo, B. K. Tay, S. Chen, W. Zhou, Z. Liu, and Z. X. Shen, Nano Lett. 15, 8155 (2015).

34 J. He, K. Hummer, and C. Franchini, Phys. Rev. B 89, 075409 (2014).

35 T. Brumme, M. Calandra, and F. Mauri, Phys. Rev B 91, 155436 (2015).

36 D. Xiao, G.-B. Liu, W. Feng, X. Xu and W. Yao, Phys. Rev. Lett. 108, 196802 (2012).

37 S. Fang, R. K. Defo, S. N. Shirodkar, S. Lieu, G. A. Tritsaris, and E. Kaxiras, Phys. Rev. B 92, 205108 (2015).

38 G. Aivazian, Z. Gong, A. M. Jones, R.-L. Chu, J. Yan, D. G. Mandrus, C. Zhang, D. Cobden, W. Yao, and X. Xu, Nat. Phys. 11, 148 (2015).

39 A. Srivastava, M. Sidler, A. V. Allain, D. S. Lembke, A. Kis, and A. Imamoğlu, Nat. Phys. 11, 141 (2015).

40 M. Koshino and T. Ando, Phys. Rev. B 81, 195431 (2010).

41 M. Nakamura, L. Hirasawa, and K. I. Imura, Phys. Rev. B 78, 033403 (2008).

42 M. Zarenia, P. Vasilopoulos, and F. M. Peeters1, Phys. Rev. B 85, 245426 (2012).

43 X. F. Wang and P. Vasilopoulos, Phys. Rev. B 72, 085344 (2005); 67, 085313 (2003).

44 M. Charbonneau, K. M. Van Vliet, and P. Vasilopoulos, J. Math. Phys. 23, 318 (1982).

45 M. Koshino and T. Ando, Phys. Rev. B 77, 115313 (2008).

46 Z. Li, and J. P. Carbotte, Phys. Rev. B 88, 045414 (2013).

47 C. J. Tabert and E. J. Nicol, Phys. Rev. B 88, 085434 (2013).

48 E. Illes and E. J. Nicol, Phys. Rev. B 94, 085434 (2016).

49 V. P. Gusynin, S. G. Sharapov, and J. P. Carbotte, J. Phys.: Condens. Matter 19, 026222 (2007); Phys. Rev. 
Lett. 98, 157402 (2007).

50 E. Illes and E. J. Nicol, Phys. Rev. B 87, 245131 (2013). 